\title{
EVALUATION OF LIVER AND LIPID PARAMETERS IN CHOLELITHIASIS
}

\author{
GURPREET KAUR GILL*, PRABHJOT KAUR
}

Department of Medical Lab Sciences, Khalsa College of Pharmacy and Technology, Amritsar, Punjab, India. Email: gurpreet_pau25@yahoo.com Received: 22 April 2019, Revised and Accepted: 03 July 2019

\section{ABSTRACT}

Objectives: The objectives of the present study were to compare the biochemical parameters in gallstones patients and controls and to study the differences in their levels in male and female patients.

Methods: The patient data were collected from the Navpreet Hospital, Amritsar. All the demographic data and ultrasonographic features were noted in a pre-structured pro forma of cholelithiasis patients. The blood sample was collected in aseptic conditions and serum biochemical parameters of liver (serum glutamic oxaloacetate transaminase [SGOT] and serum glutamic pyruvic transaminase [SGPT]) and lipids (cholesterol and triglyceride) were investigated in the patients.

Results: In this study, it was found that cholelithiasis is more prevalent in females (76\%) as compared to females (26\%). The SGOT and SGPT levels were higher in $20-40$ years age group females with a mean of $78 \pm 7.12$ and $70 \pm 5.34 \mathrm{mg} / \mathrm{dl}$, respectively. Alkaline phosphatase (ALP) level also increases in female patients with a mean of $106 \pm 5.36 \mathrm{mg} / \mathrm{dl}$. Same pattern was observed for bilirubin and lipid levels.

Conclusion: The prevalence of gallstones was found more in females than males. Serum cholesterol, triglyceride, SGOT, SGPT and ALP, and bilirubin play a major contributing role in the formation of gallstone in females. Incidence of gallstones is most often correlated with dietary factors, obesity or overweight, sedentary lifestyle, eating high fat and cholesterol diet, eating low-fiber diet, having a family history of gallstones, and having liver disease.

Keywords: Cholelithiasis, Cholesterol, Serum glutamic oxaloacetate transaminase, Serum glutamic pyruvic transaminase, Alkaline phosphatase, Bilirubin.

(C) 2019 The Authors. Published by Innovare Academic Sciences Pvt Ltd. This is an open access article under the CC BY license (http://creativecommons. org/licenses/by/4. 0/) DOI: http://dx.doi.org/10.22159/ajpcr.2019.v12i9.33694

\section{INTRODUCTION}

Cholelithiasis or gallstone disease is referred to the formation of gallstones in the gallbladder which is a very common gastrointestinal disorder [1]. Although the disease has a low mortality rate, its economic and health impact is significant due to its high morbidity. Gallstones represent a significant burden for health-care systems worldwide and are one of the most common disorders presenting to emergency room. Majority of the gallstones are caused by complex interaction of genetic and environment factors. Gallstone disease also develops in hemolytic disorders due to the solubility of the mutant proteins and hemoglobin subunit in the red blood cells [2]. In northern India, dietary factors may be responsible for formation of gallstones. Most of the gallstones are silent and asymptomatic which can further lead to cholecystitis and gall bladder cancer if untreated [3]. Migration of a gallstone into the opening of the cystic duct may block the flow of bile and contraction of gallbladder leads to the characteristics of biliary colic pain in the upper abdomen, especially in the right hypochondriac region, back pain between shoulders, nausea or vomiting, fever, heartburn, and indigestion [4].

The bile stored in gallbladder slowly release into the digestive system through common bile duct where it helps in digestion and absorption of fat [5]. After digestion, if the gallbladder is not emptied out completely, the bile that remains in the gallbladder can become saturated usually with either cholesterol or bilirubin which leads to the formation of gallstones [6]. Cholesterol supersaturation of bile together with biliary stasis predisposes to cholesterol gallstone formation [2]. Cholelithiasis can also lead to cholecystitis which is defined as a chemical or bacterial inflammation of the gallbladder that occurs due to an obstruction of the cystic duct. Obstruction to the gallbladder drainage causes distension and wall edema which may progress to venous and lymphatic obstruction, ischemia, and necrosis. In chronic cholecystitis, the gallbladder is damaged by repeated attacks of acute inflammation, usually due to gallstones and become thick walled and small [7].

Biochemical parameters get altered in gall stone patients and assessed as organ damage. Liver function test play important role in studying the functions of different cells and tissues. Aspartate transaminase (AST) and alanine transaminase (ALT) are the measures of hepatocellular damage, inflammation of biliary tract and obstruction of bile ducts. And due to hemolysis and obstruction of bile duct, bilirubin levels can be elevated and shows symptoms of jaundice [8].

The aim of the study was to investigate the changes in the serum biochemical markers of hepatic function of pathological conditions in patients with evident cholelithiasis. The increase in the inflammatory and pro-inflammatory markers in gallbladder tissue systemically affects other tissues as well. One of the important pathological indications of the presence of gallstones is the decreased hepatic function which could be due to altered cellular metabolism as a result of sustained affliction by pro-inflammatory conditions in the body.

\section{METHODS}

The present investigation was a case-control study carried out in the research laboratories of Khalsa College of Pharmacy and Technology and Khalsa Diagnostic Laboratory, Amritsar. Samples were collected from Navpreet Hospital, Amritsar. The study was carried out on diagnosed pre-operative and symptomatic patients of cholelithiasis. Symptoms included pain in upper abdomen and pain over epigastric region. Diagnosis was based on medical history of patient physical examination and ultrasound examination. Ultrasound was performed by the expert sonologists. Informed written consents were taken from every patient after informing the procedure regarding the study and samples were procured after taking ethical approval by 
the institutional ethical committee. Blood samples of a total of 50 gallstone patients were selected for the study, of which 38 were female and 12 were male. Patients who visited the outpatient department of hospital for routine check-up were served as controls. Inclusion criteria involved the male and female gallstone patients with the age group of 20-80 years of both. Exclusion criteria involved pregnant women, adolescent girl, growing children, patients of gallbladder and pancreatic carcinoma, emergency operation, patients having age $<20$ years, and non-cooperative patients. Blood sample was collected by venipuncture method. Samples were centrifuged at $3000 \mathrm{rpm}$, separated, and preserved at $4^{\circ} \mathrm{C}$ for further analysis. Laboratory parameters were investigated using ERBA and Beacon Biochemical Kits on semi-automated analyzer. The activity of serum glutamic oxaloacetate transaminase (SGOT) and serum glutamic pyruvic transaminase (SGPT) was determined by the International Federation of Clinical Chemistry (IFCC) method [9]. Alkaline phosphatase (ALP) activity was determined by the IFCC method [10]. The level of total and direct bilirubin was determined by dimethyl sulfoxide method [11]. Indirect bilirubin was calculated by subtracting the amount of direct bilirubin from total bilirubin. Serum cholesterol

Table 1: Demographic data of gallstone patients and controls under study

\begin{tabular}{llllll}
\hline \multirow{2}{*}{$\begin{array}{l}\text { Study } \\
\text { parameters }\end{array}$} & \multicolumn{2}{l}{ Gallstone patients } & & \multicolumn{2}{l}{ Normal controls } \\
\cline { 2 - 3 } \cline { 5 - 6 } \cline { 5 - 6 } & Females & Males & & Females & Males \\
\cline { 2 - 3 } \cline { 5 - 6 } & $\mathbf{n = 3 8}$ & $\mathbf{n = 1 2}$ & & $\mathbf{n = 1 8}$ & $\mathbf{n = 1 2}$ \\
\hline Age (years) & $46.4 \pm 5.88$ & $47.5 \pm 6.13$ & & $45.5 \pm 5.38$ & $48.1 \pm 5.9$ \\
Height (feet) & $5.3 \pm 0.22$ & $6.1 \pm 0.46$ & & $5.3 \pm 0.27$ & $5.9 \pm 0.36$ \\
Weight (kg) & $70.6 \pm 9.4$ & $96.2 \pm 5.80$ & & $66.6 \pm 8.66$ & $91.6 \pm 9.89$ \\
\hline
\end{tabular}

*Data are represented as mean \pm standard deviation

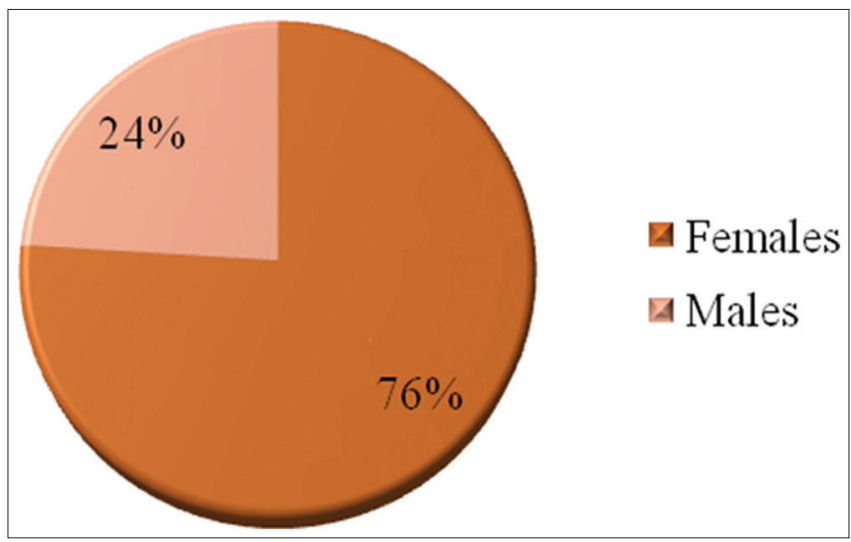

Fig. 1: Prevalence of gallstones disease in males and females and triglycerides were estimated by CHOD-PAP method [12] and GPO/POD method [13], respectively.

\section{RESULTS AND DISCUSSION}

Cholelithiasis represents a major clinical condition and it affects millions of people around the globe. The present investigation was basically a case-control study which included diagnosed and symptomatic patients of cholelithiasis.

Gallstone patients who visited hospital were investigated for their biochemical profiles and $76 \%$ of them were found to be females and $24 \%$ were male. It has been inferred that cholelithiasis disease is more prevalent in females (Fig. 1).

Mean age, height, and weight of patients included in the study were comparative to the age, height, and weight of controls, respectively (Table 1). SGOT enzyme primarily found in the liver and heart cells. SGOT enzyme was found higher in gallstone patients as compared to the healthy controls. Further, it was observed that female gallstone patients had higher SGOT levels as compared to male patients. In control group, SGOT activity in both females and males found almost similar and in the normal range. Male controls have SGOT levels significantly similar to female controls. Similarly, SGPT is an enzyme made in the liver and is released into the blood when tissues are damaged. SGPT levels were found higher in female gallstone patients as compared to male patients. Control subjects were found to have normal SGPT levels. Male controls have SGPT levels significantly similar to female controls. SGOT and SGPT levels are generally considered as measures of hepatocellular damage and due to gallstone disease liver are inflamed and damaged which simultaneously causes rise in hepatic enzymes in blood [8]. ALP levels were found extremely high in female gallstone patients as compared to males. Both males and females recruited in control group had ALP levels comparative to each other, although lower than gallstone patients. the activity of all the enzymes studied viz. SGOT, SGPT and ALP were found higher in female gallstone patients (Fig. 2).

Bilirubin levels were found higher in gallstone patients as compared to controls under investigation. The total, direct, and indirect bilirubin levels were found higher in females as compared to males (Fig. 3). The normal range of total bilirubin found to be up to $1.0 \mathrm{mg} / \mathrm{dl}$ and direct bilirubin up to $0.3 \mathrm{mg} / \mathrm{dl}$ in healthy adults. Bilirubin is one of the indicators of gallstones but not as reliable as ALP. The degree of hyperbilirubinemia reflects the degree of liver dysfunction affecting both nutrition and reticuloendothelial functioning.

Gallstone patients had higher cholesterol levels as compared to controls, however, in female patients, cholesterol levels were found low as compared to male patients (Fig. 4). Triglyceride levels were found higher in female gallstone patients as compared to male patients.

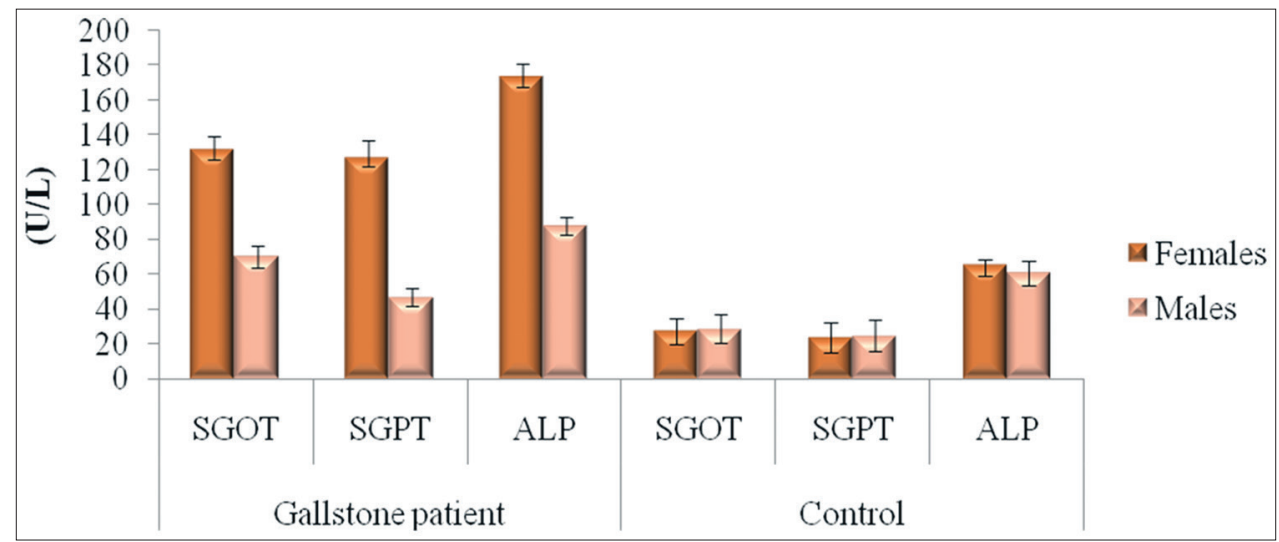

Fig. 2: Levels of liver enzymes in male and female patients of cholelithiasis 


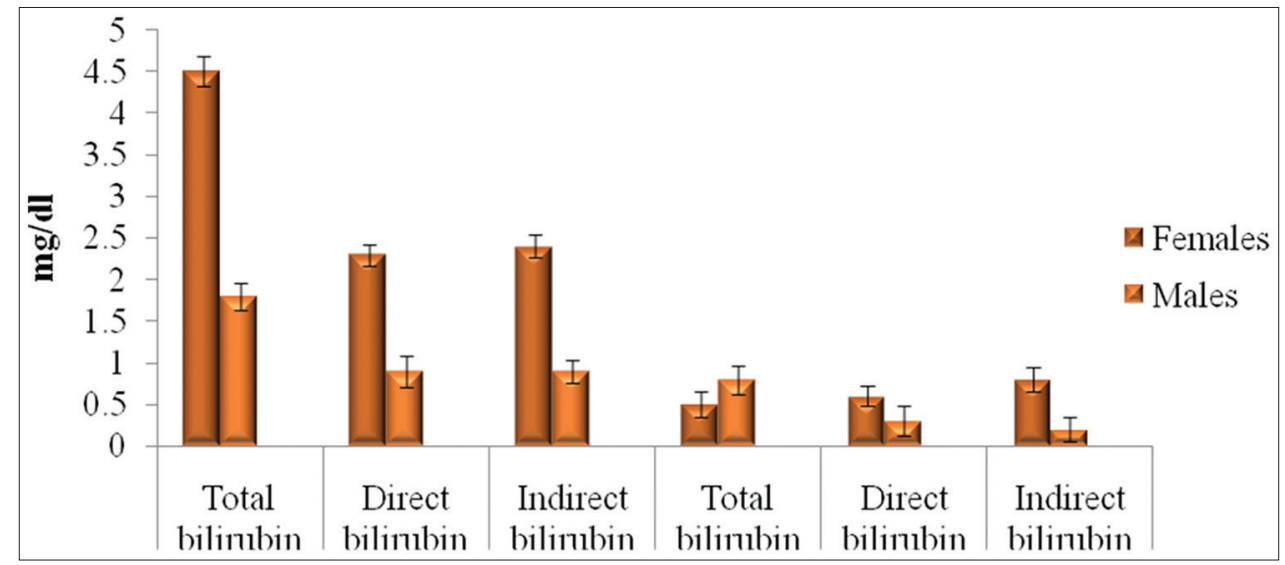

Fig. 3: Levels of total, direct, and indirect bilirubin in male and female patients of cholelithiasis

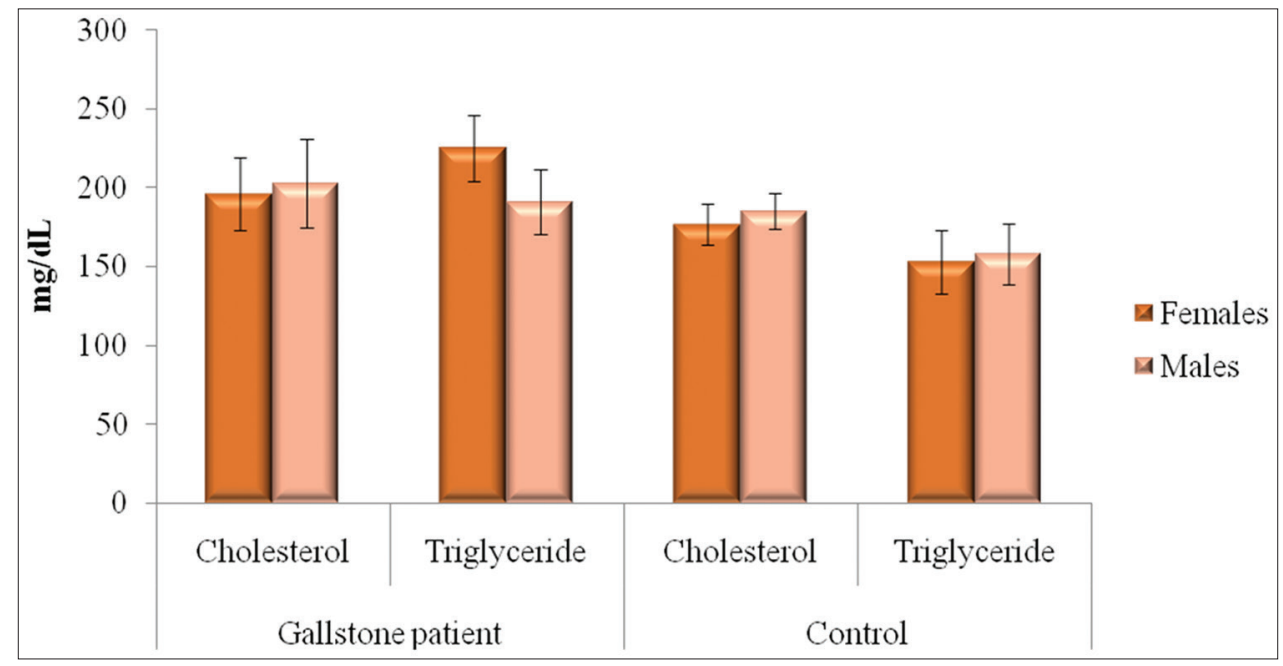

Fig. 4: Levels of cholesterol and triglyceride in male and female patients of cholelithiasis

Control subjects were found to have normal triglyceride levels. Male controls have triglyceride levels significantly similar to female controls. High levels of serum lipids have been found associated with gallstone disease. In reproductive-aged women, risk is higher as compared to non-reproductive age, may be due to elevated estrogen levels, which increase cholesterol excretion in bile by causing its supersaturation with cholesterol [14]. Free cholesterol increased steroid synthesis, this, in turn, decreased the production of bile acids and, hence, of bile salts which kept the cholesterol soluble in bile. Decreased concentration of bile salts is responsible for their precipitation of bile salts, leading to the formation of gallstones [15]. This suggested that altered serum lipid profile is a possible consequence of the presence of gallstones, especially through biliary obstruction. It can be suggested that physical activity and avoiding fried foods would result in the prevention of gallstones prevalence.

Triglycerides accumulated in hepatocytes in fatty liver condition, triggered inflammatory responses that lead to the leakage of liver enzymes into the bloodstream. Gallbladder did not empty normally, thus causing bile accumulation which precipitates gallstones. Long list of investigations makes the diagnostic pathway complex and expensive, but good clinical history can be worked out as the best predictor of gallstones. Abnormal liver function tests were most common in patients with gallstones. Raised level of ALP has emerged as the most reliable predictor of gallstones. In our study, subjects were predominantly females and ALP levels concerned with injury of biliary tract and increase in its level might be due to increased bone turnover or simultaneous formation of osteoid in females [16].
The diseased gallbladder is one of the most common specimens submitted to the surgical pathology laboratory in North India. The ratio of male-to-female in gallstone patients has also been determined to be 1:3, affected with altered lipid levels. They found the higher level of triglycerides in females and higher cholesterol in males [17]. Gender difference in lipid levels might be due to the dietary differences in this particular region and should be studied further. Gender differences in lipid patterns of gallstone patients were determined [18]. Lipid metabolism is also associated with gallstone formation. Further, the positive association between gallstone disease and coronary heart disease is determined [19]. Obesity in females may also be estimated as a gender-specific risk factor for gallstone formation as it has been established that high body mass index can lead to cholelithiasis [20].

\section{CONCLUSION}

Studying the pathogenesis and physiology of cholelithiasis may allow expanding the indication for therapeutic treatment of disease and reducing the number of patients who undergo surgical treatment. Elevated serum cholesterol, triglyceride, SGOT, SGPT, and bilirubin play major contributing role in the formation of gallstones in females. Females were found more prone to develop gallstones as compared to males in the present study.

\section{ACKNOWLEDGMENT}

Authors are thankful to Dr. R. K. Dhawan, Khalsa College of Pharmacy and Technology, for providing the necessary facilities and funds for the 
project. Further, authors are thankful to Navpreet Hospital, Amritsar, for permitting the collection of required clinical samples for the study.

\section{CONFLICTS OF INTEREST}

Declared none.

\section{AUTHORS' CONTRIBUTIONS}

Prabhjot Kaur collected the clinical samples and carried out biochemical investigations. Gurpreet Kaur Gill drafted the manuscript. All authors read and approved the final manuscript.

\section{REFERENCES}

1. Charles F, Bruncicardi M, Thai H. Gallbladder and the extrahepatic biliary system. Schwartz Princ Surg 2009;8:1135-67.

2. Abdullah UY, Jassim HM, Baig AA, Khorsheed RM, Al-Khayat AM, Sulong AF, et al. Gallstones in patients with inherited haemolytic diseases. Int J Pharm Pharm Sci 2015;7:9-15.

3. Heiss G, Tamir I, Davis CE, Tyroler HA. The circulatory system. Circ J 1980;61:302.

4. Pani JP, Pandey MS, Pani DS, Maderakar MN, Katti HK. Estimation of predominate histologic alterations in cholecystitis and cholelithioasis of human gallbladder an analytical and statistical study through the approach of routine histochemistry. J Dent Med Sci 2013;6:35-43.

5. Meilstrup JW, Hopper KD. Imaging atlas of the normal gallbladder and its variants. J Gastroenterol Hepatol 1991;157:1205-08.

6. Guyton AC, John E. Assessment of gallstone predictor comparative analysis of ultrasonographic and biochemical parameters. Textbook Med Physiol 2005;11:802-4.
7. Nahrwold DL. The biliary system. In: Textbook of Surgery: The Biological Basis of Modern Surgical Practice. Vol. 15. USA: W.B. Saunders Company; 1997. p. 1117-35.

8. Ahmad NZ. Routine testing of liver function before and after elective laparoscopic cholecystectomy: Is it necessary? JSLS 2011;15:65-9.

9. Wallnofer H, Schmidt E, Schmidt FW. Determination of SGOT and SGPT by IFCC method. J Clin Biochem 1986;24:497-507.

10. Bessey OA, Brock MJ. Determination of ALP. Biol Chem 1946;164:321.

11. Jendrarrisk L, Grof P. Determination of bilirubin by DMSO Method. Clin Biochem 1983;2:29.

12. Roeschlau P, Bernt E, Gruber WA. Determination of cholesterol. Clin Biochem 1974;12:266.

13. Bergmeyer M, Schetter G. Determination of triglyceride by GPO-POD method. Clin Chem 1975;10:25.

14. Reshetnyak VI. Concept of the pathogenesis and treatment of cholelithiasis. World J Hepatol 2012;4:18-34.

15. Channa NA. Hypercholesterolemia in adults population of Pakistan. Pak Armed Forces Med J 2008;58:197.

16. Bose SM, Mazumdar A, Prakash VS, Kocher R, Katariya S, Pathak CM, et al. Evaluation of the predictors of choledocholithiasis: Comparative analysis of clinical, biochemical, radiological, radionuclear, and intraoperative parameters. Surg Today 2001;31:117-22.

17. Saraya A, Irshad M, Gandhi BM, Tandon RK. Plasma lipid profile in gallstone patients from North India. Trop Gastroenterol 1995;16:16-21.

18. Tandon RK, Saraya A, Paul S, Kapur BM. Dietary habits of gallstone patients in Northern India. J Clin Gastroenterol 1996;22:23-7.

19. Grigor'eva IN, Maliutina SK, Voevoda MI. Role of hyperlipidemia in cholelithiasis. Eksp Klin Gastroenterol 2010;(4):64-8.

20. Völzke H, Baumeister SE, Alte D, Hoffmann W, Schwahn C, Simon P, et al. Independent risk factors for gallstone formation in a region with high cholelithiasis prevalence. Digestion 2005;71:97-105. 